\title{
STEADY STATE DIFFUSION PROCESSES UNDER TURBULENT CONDITIONS. GALLIUM AND ALUMINIUM CASES.
}

\author{
JÁN BUDAJ, MILAN ZBORIL, JURAJ ZVERKO, JOZEF ŽIŽŇOVSKÝ \\ Astronomical Institute, Slovak Academy of Sciences, \\ 05960 Tatranská Lomnica, Czech and Slovak Federal Republic \\ JOZEF KLAČKA \\ Department of Astronomy and Astrophysics \\ Faculty for Mathematics and Physics Comenius University \\ Mlynská dolina, 84215 Bratislava, Czech and Slovak Federal Republic
}

\begin{abstract}
The stationary state of the element stratification under appropriate turbulence is investigated. Equation of the stationary state is derived and solved under several simplifications. Cases of $\mathrm{Ga}$ and $\mathrm{Al}$ are studied. Al is predicted to be underabundant, but the abundance is rising with decreasing effective temperatures of the stars. Different results obtained using two methods of finding the stationary Ga stratification are indicated.
\end{abstract}

\section{INTRODUCTION}

Stratification of the atmospheres of Ap stars occurs as a consequence of accumulation of atoms due to diffusive processes controlled by the radiative acceleration (e.g., Michaud 1970). The calculations of the depth- and time-dependent abundances are not easy to carry out. Quantities used ( $g f-$ values...) are known within a few percent; the characteristic time of such processes is $10^{3}$ years and the typical diffusion velocities are $\sim \mathrm{cms}^{-1}$, unknown time dependent turbulence may take place. Perhaps an easier way could be finding a possible final state when stability is reached. Alecian and Artru (1988) calculated the stratification of Ga under the assumption of zero diffusion velocities. Bergeron et al.(1988) made similar computations for other species. However, a more general equilibrium state can be reached, i.e., the fluxes of particles of a certain element are constant in both time and depth.

\section{EQUATION OF THE STATIONARY STATE}

The above-made assumption can be expressed as

$$
\text { const. }=\sum_{i} n_{i}(r) v_{i}(r)
$$

where $n_{i}$ is the number density of $i$-th ion, $v_{i}$ is its diffusion velocity, and a summation takes place through all important ions at a given depth $r$. We can choose const. as a flux of the element at the bottom of the atmosphere, having only two free parameters 
$n, \frac{\partial n}{\partial r}$. Including the diffusion equation (see Aller and Chapman, 1960) one can write (all the quantities in the following considerations are supposed to be depth-dependent):

$$
\begin{array}{r}
\text { const. }=\sum_{i} n_{i} D_{i}\left[-\left(1+\frac{D_{T}}{D_{i}}\right) \frac{1}{c_{i}} \frac{\partial c_{i}}{\partial r}+\right. \\
+\underbrace{\frac{2 W-Z_{i}-1}{p} \frac{\partial p}{\partial r}+\frac{2.54 Z_{i}^{2}+0.805\left(W-Z_{i}\right)}{T} \frac{\partial T}{\partial r}}_{T P_{i}}+\frac{m}{k T} g_{i}^{r a d}\left(c_{i}\right)]
\end{array}
$$

here $c_{i}, W, Z_{i}, p, k, T, g_{i}^{r a d}$ are the $i$-th ion abundance, the atom mass number, the ion charge, the pressure, the Boltzmann constant, the temperature as well as the radiative acceleration of the $i$-th ion, respectively. The term $\left(1+\frac{D_{T}}{D_{i}}\right)$ takes into account turbulence, $D_{T}, D_{i}$ being the turbulent and ion diffusion coefficients. After some simplifications in Equation (2) we obtain for the element abundance $c$ :

$$
\frac{\partial c}{\partial r}+c \underbrace{\frac{\sum_{i}\left(D_{i}+D_{T}\right) \frac{\partial f_{i}}{\partial r}-f_{i} D_{i}\left(T P_{i}+\frac{m}{k T} g_{i}^{r a d}\left(c_{i}\right)\right)}{\sum_{i}\left(D_{i}+D_{T}\right) f_{i}}}_{\alpha(c)}+\underbrace{\frac{\text { const. }}{n_{H} \sum_{i}\left(D_{i}+D_{T}\right) f_{i}}}_{\beta}=0
$$

where $f_{i}=\frac{n_{i}}{n}$, and $n, n_{H}$ are the element and all atoms number densities, respectively. In the case where there are no saturated lines of the element investigated equation (3) can be further simplified because of independence of $g_{i}^{r a d}$ on the abundance, thus

$$
\frac{\partial c(r)}{\partial r}+c(r) \alpha(r)+\beta(r)=0
$$

The above introduced equations may yield a very strong stratification for a number of normally abundant elements. To get into agreement with observations we have supposed presence of a slight, depth-dependent turbulence having a growing value with decreasing optical depth, which is analogous, to some extent, to microturbulence behaviour in cool Ap star as indicated in Zboril (1992). One could find the turbulence by means of fitting the observed abundances of two elemens. We supposed the free parameters at the bottom of the atmosphere to be: $c_{0}=$ solar, $\frac{\partial c}{\partial r}=0$ but one could take them from time-dependent calculations (more precise in deep layers). The radiative accelerations were computed using the same formula as in Budaj et al. (1991). The partition functions are after Irwin (1981), being of a sufficient accuracy for $T<16000 \mathrm{~K}$. Radiative fluxes calculations were based on Milne's approximation. The opacities computed using a modified code SYNSPEC developed by Hubený (1987), represent, in addition to opacities in the lines of interest the others: Rayleigh scattering, $\mathrm{H}^{-}$, bound-free as well as all contributed line opacities. All computations were done assuming LTE. That is why results for $\log ($ ros.opt.depth $)<-2$ are only indicative. CGS units were used.

\section{$\underline{\text { ALUMINIUM }}$}

Aluminium has a tendency to be underabundant in $\mathrm{Mn}, \mathrm{Sr}, \mathrm{Eu}-\mathrm{Cr}-\mathrm{Sr}$ stars, is of a normal abundance in Si stars, and enhanced (up to 10 times when compared with the Sun) in Am stars (Hack and Struve 1970). Sadakane et al.(1983) found that Al is definitely underabundant by $1.0 \mathrm{dex}$ in $\mathrm{Hg}-\mathrm{Mn}$, Si and He-weak stars except for five coolest $\mathrm{Hg}$-Mn 


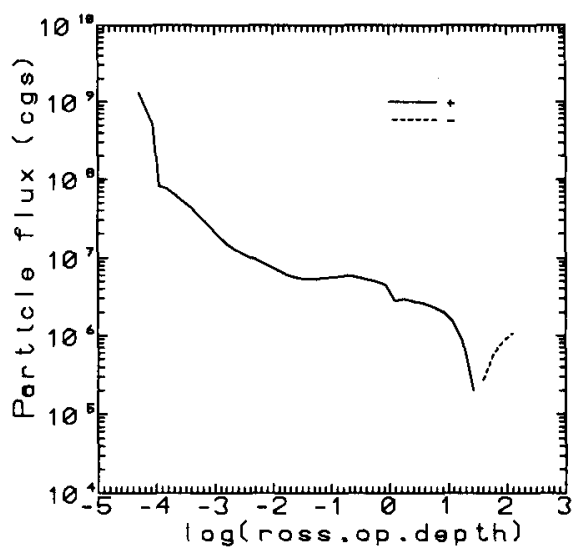

Figure 1: Al-particle flux, + particles leave the atmosphere (radiative acceleration is dominant), - particles plunge (gravitational settling is dominant)

stars (only with a slight underabundance by $0.5 \mathrm{dex}$ ). Computations have been done using the Kurucz model $T_{\text {eff }}=12000 K, \log g=4.0(\mathrm{cgs})$. The line list (Kurucz and Peytremann 1975) contains several thousands of Al lines. That is why we have had to select strongly absorbing lines only, gathering them subsequently. Radiative acceleration

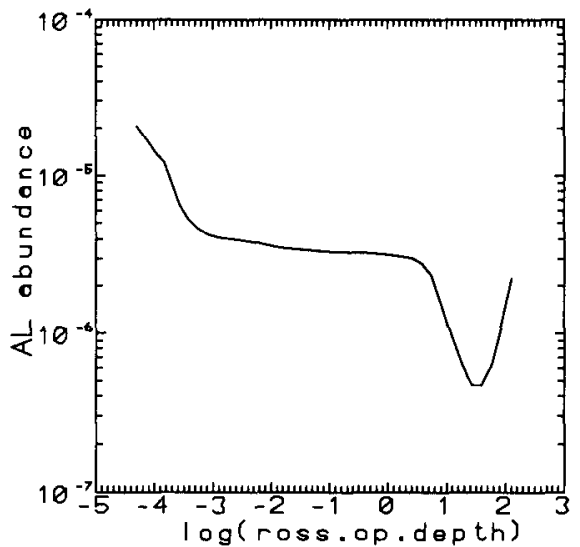

Figure 2: Example of the Al stratification. The unreal behaviour in outer part of the atmosphere is caused by unjustified assumption of zero particle flux everywhere. On the other side this assumption seems to be more justified just in deep areas where gravitational settling is dominant .

of Al IV ion has been found to be negligible. In the Fig.1 the particle flux is plotted with solar homogeneous abundances. Solution of the steady state equation under the assumption of everywhere zero flux only seems to be physicaly justified in deep layers of the atmosphere (see Fig.2; the same assumption when applied to higher parts of the atmosphere leads clearly to unphysical situation there in the sense that without taking into account turbulence Al would be nearly depleted from the atmosphere within about $10^{5}$ years). Here the abundance falls rapidly down and its gradient is sensitive to the 
turbulence. This may explain large underabundances in hotter Ap stars, small underabundances in the five coolest $\mathrm{Hg}-\mathrm{Mn}$ stars and the tendency of $\mathrm{Al}$ to be an abundant element in cool Am stars because of comming out of the convection zone and plunging of areas where gravitational settling is dominant (radiative acceleration of Al IV should still be negligible) with decreasing effective temperature. However, only detailed calculations of diffusion velocities - we use only approximative expresions for diffusion coefficients as well as fluxes of particles and abundance-dependent radiative accelerations enable us to shed a substantial light on this problem.

\section{GALLIUM}

Gallium is famous for large overabundances by more than 3 dex in Hg-Mn stars. Alecian (1987) studied this for a model of the atmosphere with $T_{\text {eff }}=12000 \mathrm{~K}, \log \mathrm{g}=4.0$. We used radiative accelerations from his paper under a rough assumption of their abundance independence. In the Fig. 3 we found out the stratification that would yield a

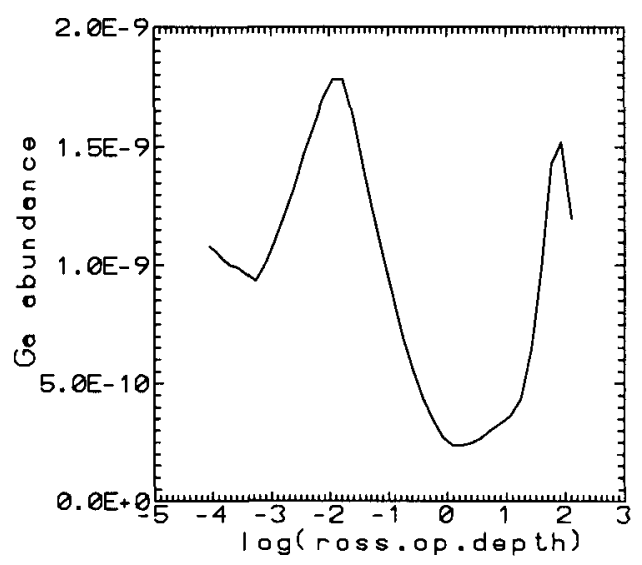

Figure 3: Ga stratification computed using iteration method (see text)

constant flux of $\mathrm{Ga}$ atoms (without turbulence) using the following iteration formula: $c_{i}(r)=c_{i-1}(r) \frac{f l u x_{0}}{f l u x_{i-1}(r)}$ where flux $x_{0}$ means particle flux at the bottom of the atmosphere, $f l u x_{i-1}(r)$ particle flux at depth $r$ is computed for the abundances $c_{i-1}(r)$. Iterations will stop when $\frac{f l u x_{0}}{f l u x_{i-1}(r)} \sim 1$. Analogous methods are used from time to time to determine an element stratification in equilibrium (zero particle flux) and the term $\frac{1}{c} \frac{\partial c}{\partial r}$ is supposed to be negligible. Here, the starting conditions may not be valid at the bottom $c_{0}, \frac{\partial c}{\partial r}$. In Fig. 4 the Ga abundances were obtained solving the steady state equation with Runge-Kutta method under the assumption of similar turbulence behaviour like in Al cases.

\section{ACKNOWLEDGEMENTS}

We would like to thank Dr. Alecian for providing us with the Ga lines data, and to Dr. Komžík and Dr. Saniga for their help in preparing this paper. 


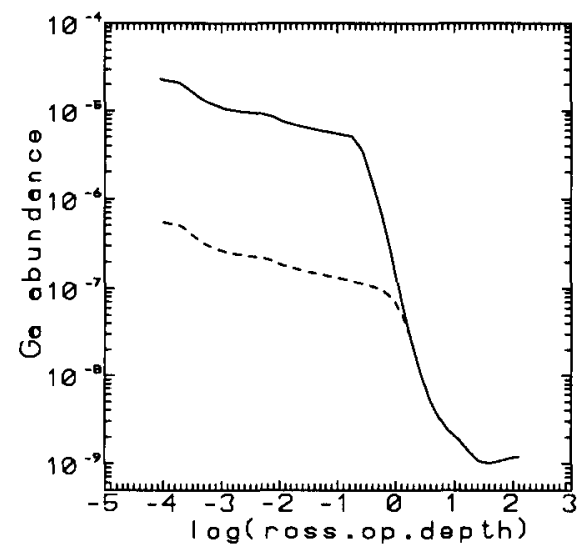

Figure 4: Ga stratification computed using Runge-Kutta method with turbulence diffusion coefficients $D_{T}$ increasing from $3.10^{5}$ at the bottom to $10^{10}$ at the top of the atmosphere, dashed line - $D_{T}$ is three times larger in the central part of the atmosphere

\section{REFERENCES}

Alecian, G.1987, Astr. Ap., 60, 153

Alecian, G., Artru, M.-C. 1988, in: The impact of very high $S / N$ spectroscopy on stellar physics. 159

Aller, L.H., Chapman, S. 1960, Ap. J., 132, 461

Bergeron, P., Wesemael, F., Michaud, G., Fontaine, G. 1988, Ap. J., 332, 964

Budaj, J., Zboril, M., Zverko, J. 1992, in: Lecture Notes in Physics, 401, 210

Hack, M., Struve, O. 1970, in: Stellar Spectroscopy - Peculiar Stars (Osservatorio Astronomico Di Trieste)

Hubený, I. 1987, Scient. and Techn. Rep. Astr. Inst. Czechoslov. Acad. Sci. No. 40

Irwin, A. W. 1981, Ap. J. Suppl., 45, 621

Kurucz, R. L., Peytremann, E. 1975, SAO Spec. Report 362

Kurucz, R. L. 1979, Center for Ap. Reprint Series No. 1050

Michaud, G. 1970, Ap. J., 160,641

Sadakane, K., Takada, M., Jugaku, J. 1983, Ap. J., 274, 261

Zboril, M. 1992, in: Proc. International Conference "Stellar Magnetism", ed. Yu. V. Glagolevski, in press 